\title{
Surgical Site Infection - Relevance of Preoperative Assessment of Albumin and Cholesterol
}

\author{
Authors \\ Dr Nowshad.M ${ }^{1}$, Dr C.V.Rajendran ${ }^{2 *}$, Dr Jameela Beevi ${ }^{3}$ \\ ${ }^{1}$ Associate Professor, Surgery, Travancore Medical College, Kollam \\ ${ }^{2}$ Additional Professor, Department of Surgery, Government Medical College, Trivandrum \\ ${ }^{3}$ Consultant Surgeon, Government Hospital, Kottarakkara \\ *Corresponding Author \\ Dr C.V.Rajendran \\ Additional Professor, Department of Surgery, Government Medical College, Trivandrum
}

\begin{abstract}
Background: Surgical site infection is a serious problem affecting about $2 \%$ of all surgical procedure. Advances in surgical sciences and care has not reduced the prevalence to such an extent. Various risk factors like Age, nutritional status, diabetes, smoking, obesity, altered immune status were reported in literature. There are a few reports in the literature about the role for measurements of preoperative albumin and cholesterol. However there is a lack of such studies in our settings. We set out to study whether there is an association between the preoperative levels of albumin and cholesterol with postoperative surgical site infection.

Methods: We conducted this prospective cohort study in patients who underwent various surgeries in our institution after getting informed consent. Apart from the basic demographic profile, preoperative levels of albumin and cholesterol were measured along with preoperative, anesthetic and perioperative details. All statistical analysis was done in $R$ statistical software.

Results: The mean $\pm S D$ age of patients was $53.1 \pm 18.1$ years (range 10-92 years) and $15.5 \%$ developed nosocomial infection. Hypoalbuminemia was present in 87(33\%)of the patients. Hypoalbuminemia was found to be associated with SSI with a relative risk of 1.98 and confidence interval of 1.07 to 3.61. High cholesterol levels were found in 87 patients with 3(1\%) only having SSI.

Discussion: In this study, we observed that there was a positive association between Hypoalbuminemia and SSI which was statistically significant. Hence routine measurement of serum albumin and cholesterol at the time of admission will help in proper optimization of surgical patients so that potential SSI could be reduced.
\end{abstract}

\section{Introduction}

Surgical site infection continues to be one of the major causes of nosocomial infections. It accounts for to about $15-20$ percent of all nosocomial infections. In approximately $2 \%$ of all surgical procedures, surgical site infection can pose a serious complication that warrants attention to the control of this entity ${ }^{1}$. Advances in infection control practice like better operation theatre, ventilator, better surgical technique and availability of more powerful antibiotics aim to reduce the prevalence of this potentially deadly 
and debilitating complication. However despite all advances in perioperative care, modern theater set up, surgical site infection remains a threat to surgical patients ${ }^{2}$.

Various risk factors are associated with development of surgical site infection. Age, nutritional status, diabetes, smoking, obesity, altered immune status are some of the risk factors associated with surgical site infections ${ }^{3-5}$. Many preoperative routines and preoperative factors like shaving, skin preparation, operative room ventilation and drain also contribute to the development of $\mathrm{SSI}^{6-8}$.

$\mathrm{S}$. albumin level has a role in surgical site infection $^{9-12}$. A few studies have reported a relationship between low serum albumin level and high cholesterol level in surgical site infection, length of hospital stay and death and is reported to be one of the major causes of morbidity and mortality among hospitalized patients ${ }^{9,13-15}$. However. There are no such studies done in our settings. Moreover available evidences are contradictory in nature. Our hypothesis was that preoperative levels of albumin and cholesterol are associated with the susceptibility for surgical site infections.

We conducted this study to find out the relation between preoperative serum albumin and cholesterol level and risk of surgical site infection.

\section{Materials and Methods}

This prospective cohort study was conducted in the year 2008 in the department of surgery government medical college trivandrum.

We conducted this study in concordance with the declaration of Helsinki. Informed consents were obtained from all patients participating in the study. Confidentiality of the subjects were maintained before during after the study.

Patients who underwent surgery in surgical department were chosen for the study. Patients in all units including surgical gastroenterology were chosen in the period June 2008- December 2008. These patients admitted to wards for less than 1 day were excluded. Random samples were taken from patients undergoing commonly performed surgeries in our institution. The surgical procedures included in the study were head and neck surgery, breast surgery, inguinal hernia surgery, appendectomy and colorectal surgery.

Preoperative shaving was done in all cases. Povidone solution was painted all over the surgical site. Strict aseptic precautions were adopted throughout the surgery. In most of the surgeries a suction drains tube were used. At the end of surgery conventional dressing were applied. Serum samples obtained analysed for total cholesterol and albumin in the same laboratory.

The duration of procedure in assessed from anesthesia note - The patients were monitored till the date of discharge. Any surgical site infection seen was recognized. The preoperative $S$. Albumin, S. Cholesterol length of hospital stay duration of surgery is considered for this study and its relation to surgical site infection were obtained. Antimicrobial prophylaxis, treatment and type, culture and sensitivity of organism were not considered.

An operative definition of surgical site infection was used for an objective case selection and to avoid any bias. We followed the definition as given by $\mathrm{CDC}^{16}$.

A proforma was prepared incorporating the levels of S. albumin, S. Cholesterol length of stay and death duration of procedure for each surgery. All relevant information including basic demographic variables were collected and later abstracted to an excel based database for analysis.

For every serum albumin level and cholesterol fractions the prevalence of infection and mortality is calculated by dividing the number of events by number of patients that category.

Length of hospital stay distribution was normalized by variant of transformation. A linear regression analysis was developed to detect independent prediction of length by analysis of variants. Relative risk for various associations were calculated. All statistical analysis was done in R statistical software. 


\section{Results}

The mean \pm SD age of patients was $53.1 \pm 18.1$ years (range 10-92 years) and $15.5 \%$ developed nosocomial infection the geometric average LOS was 7 days (inter quartile range 4-10 days).

Hypoalbuminemia was present in $87(33 \%)$ of the patients and normal albumin was reported in $174(67 \%)$ of the participants. High cholesterol levels were found in 87 patients out of which only $3(1 \%)$ had SIS.

Hypoalbuminemia was found to be associated with SSI with a relative risk of 1.968 with a confidence interval of 1.071 to 3.616 (figure 1).

Figure 1: Association between preoperative albumin and surgical site infection.

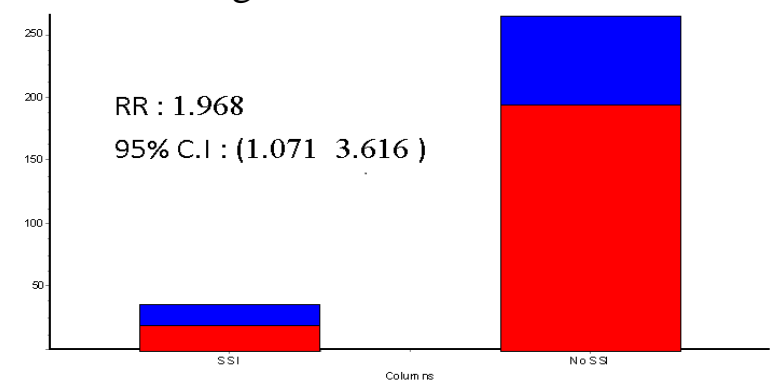

Albumin level showed in the crude analysis a clear and significant trend with nosocomial infection risk. As albumin level decreased the role of infection increased. The same trend was observed after adjusting for SENIC index, the American society of Anaesthesiologists score, age and cholesterol level. Additional risk factors did not change the output. However as in the case of albumin level, there is an increase in SSI with high cholesterol level not as in low albumin with a relative risk of 1.2 with a confidence interval of 0.2934 to 4.907 (figure 2).

Figure 2: Association between preoperative cholesterol and surgical site infection.

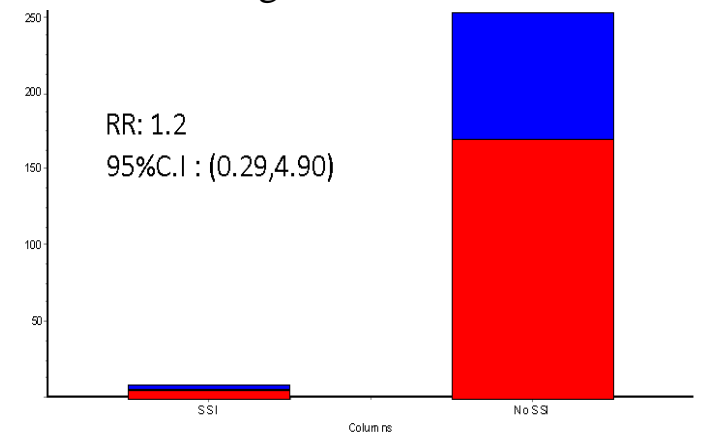

To study the relationship with LOS, a step wise linear regression model was developed. The variable included in the model were S. Albumin level, S. Cholestrol level,. Nosocomial infection, age and Duration of operation. The Association between LOS and quartiles of serum albumin and S. Cholestrol level fractions are obtained. Patients in lowest quartiles of serum albumin and High cholesterol level showed a clearly higher LOS.

Another interesting finding we observed was that abdominal surgeries increased risk of infection due to low albumin level. However there was not such relationship with cholesterol levels.There is only one death in study and control group. There is no relation with death and albumin level and cholesterol level.

\section{Discussion}

In this study, we aimed to find out the effect of preoperative albumin and cholesterol on the chance of developing surgical site infections. Our study has shown a positive association between hypoalbuminemia and SSI which was statistically significant. However hypercholesterolemia failed to show a statistically significant association between this and SSI.

In our study the relative risk was 1.968 with a confidence interval of 1.07 to 3.61 . This is in consistent with studies in the literature like Neumayer et all ${ }^{17-22}$. Englishman et al also showed that there is a positive relation between preoperative albumin level and morbidity in post operative patients ${ }^{23}$. Univariate analysis by Lee et al also showed similar association ${ }^{24}$. This points towards the importance of preoperative assessment of the patient. Lower levels of albumen needs to be evaluated and remedial measures to be started. Hypoalbuminemia could be a reflection of the nutritional status of the patient. However other reasons for the low albumin needs to be investigated. This calls for a preoperative optimization of all patients waiting for surgery except those who need immediate intervention.

In our study, there was no statistically significant association between hypercholesterolemia and 
SSI. The relative risk was 1.2 but the confidence interval was 0.2934 to 4.907 . This results corresponds to the effect size reported in literature $^{25,26}$.

One of the limitations of our study is the low sample size. It may be the reason for the failure to detect any association between cholesterol and SSI. All known confounders were not tackled in our study. Future studies with good design and with enough power could unearth potential associations with clinical relevance.

According to results the routine measurement of serum albumin and cholesterol at hospital admission is recommended. An additional advantage of including there measurements in the hospital routine is potential improvement in control of cardiovascular and renal diseases by early detection

\section{Reference}

1. de Lissovoy G, Fraeman K, Hutchins V, Murphy D, Song D, Vaughn BB. Surgical site infection: incidence and impact on hospital utilization and treatment costs. American journal of infection control 2009; 37(5): 387-97.

2. Burke JP. Infection control--a problem for patient safety. The New England journal of medicine 2003; 348(7): 651.

3. Guo Sa, DiPietro LA. Factors affecting wound healing. Journal of dental research 2010; 89(3): 219-29.

4. Owens C, Stoessel K. Surgical site infections: epidemiology, microbiology and prevention. Journal of Hospital Infection 2008; 70: 3-10.

5. Nagachinta T, Stephens M, Reitz B, Polk BF. Risk factors for surgical-wound infection following cardiac surgery. Journal of Infectious Diseases 1987; 156(6): 967-73.

6. Prabhu R. A STUDY ON SURGICAL SITE INFECTIONS. Int J Modn Res Revs 2014; 2(10): 333-5.

7. Thiele RH, Huffmyer JL, Nemergut EC. The "six sigma approach" to the operating room environment and infection. Best Practice \& Research Clinical Anaesthesiology 2008; 22(3): 537-52.

8. Anderson DJ, Kaye KS. Staphylococcal surgical site infections. Infectious disease clinics of North America 2009; 23(1): 5372.

9. Delgado-Rodríguez M, Medina-Cuadros M, Gómez-Ortega A, et al. Cholesterol and serum albumin levels as predictors of cross infection, death, and length of hospital stay. Archives of surgery 2002; 137(7): 805-12.

10. Cheadle WG. Risk factors for surgical site infection. Surgical infections 2006; 7(S1): s7-s11.

11. Wakefield C, Carey P, Foulds S, Monson $\mathrm{J}$, Guillou P. Changes in major histocompatibility complex class II expression in monocytes and $\mathrm{T}$ cells of patients developing infection after surgery. British journal of surgery 1993; 80(2): 205-9.

12. Gibbs J, Cull W, Henderson W, Daley J, Hur K, Khuri SF. Preoperative serum albumin level as a predictor of operative mortality and morbidity: results from the National VA Surgical Risk Study. Archives of surgery 1999; 134(1): 36-42.

13. Canturk N, Canturk Z, Okay E, Yirmibesoglu O, Eraldemir B. Risk of nosocomial infections and effects of total cholesterol, HDL cholesterol in surgical patients. Clinical Nutrition 2002; 21(5): 431-6.

14. Delgado-Rodríguez M, Medina-Cuadros M, Martínez-Gallego G, Sillero-Arenas M. Total cholesterol, HDL-cholesterol, and risk of nosocomial infection: a prospective study in surgical patients. Infection Control \& Hospital Epidemiology 1997; 18(01): 9-18.

15. Chien J-Y, Jerng J-S, Yu C-J, Yang P-C. Low serum level of high-density lipoprotein cholesterol is a poor prognostic factor for severe sepsis. Critical care medicine 2005; 33(8): 1688-93. 
16. Horan TC, Gaynes RP, Martone WJ, Jarvis WR, Emori TG. CDC definitions of nosocomial surgical site infections, 1992: a modification of CDC definitions of surgical wound infections. American journal of infection control 1992; 20(5): 271-4.

17. Neumayer L, Hosokawa P, Itani K, ElTamer M, Henderson WG, Khuri SF. Multivariable Predictors of Postoperative Surgical Site Infection after General and Vascular Surgery: Results from the Patient Safety in Surgery Study. Journal of the American College of Surgeons 2007; 204(6): 1178-87.

18. Haridas M, Malangoni MA. Predictive factors for surgical site infection in general surgery. Surgery 2008; 144(4): 496-503.

19. Detsky AS, Baker JP, O'Rourke K, et al. Predicting Nutrition-Associated Complications for Patients Undergoing Gastrointestinal Surgery. Journal of Parenteral and Enteral Nutrition 1987; 11: 440-6.

20. JONES CM. POSTOPERATIVE NUTRITIONAL EDEMA. Archives of Surgery 1933; 27: 159.

21. Mullen JL. Consequences of Malnutrition in the Surgical Patient. Surgical Clinics of North America 1981; 61: 465-87.

22. Rich MW, Keller AJ, Schechtman KB, Marshall WG, Kouchoukos NT. Increased complications and prolonged hospital stay in elderly cardiac surgical patients with low serum albumin. The American Journal of Cardiology 1989; 63: 714-8.

23. Engelman DT, Adams DH, Byrne JG, et al. Impact of body mass index and albumin on morbidity and mortality after cardiac surgery. The Journal of Thoracic and Cardiovascular Surgery 1999;118:866-73.

24. Lee DH, Kim SY, Nam SY, Choi S-H, Choi JW, Roh J-L. Risk factors of surgical site infection in patients undergoing major oncological surgery for head and neck cancer. Oral Oncology 2011; 47: 528-31.

25. Olsen MA, Lock-Buckley P, Hopkins D, Polish LB, Sundt TM, Fraser VJ. The risk factors for deep and superficial chest surgical-site infections after coronary artery bypass graft surgery are different. The Journal of thoracic and cardiovascular surgery 2002; 124(1): 136-45.

26. Lu JCY, Grayson AD, Jha P, Srinivasan AK, Fabri BM. Risk factors for sternal wound infection and mid-term survival following coronary artery bypass surgery. European Journal of Cardio-Thoracic Surgery 2003; 23: 943-9. 